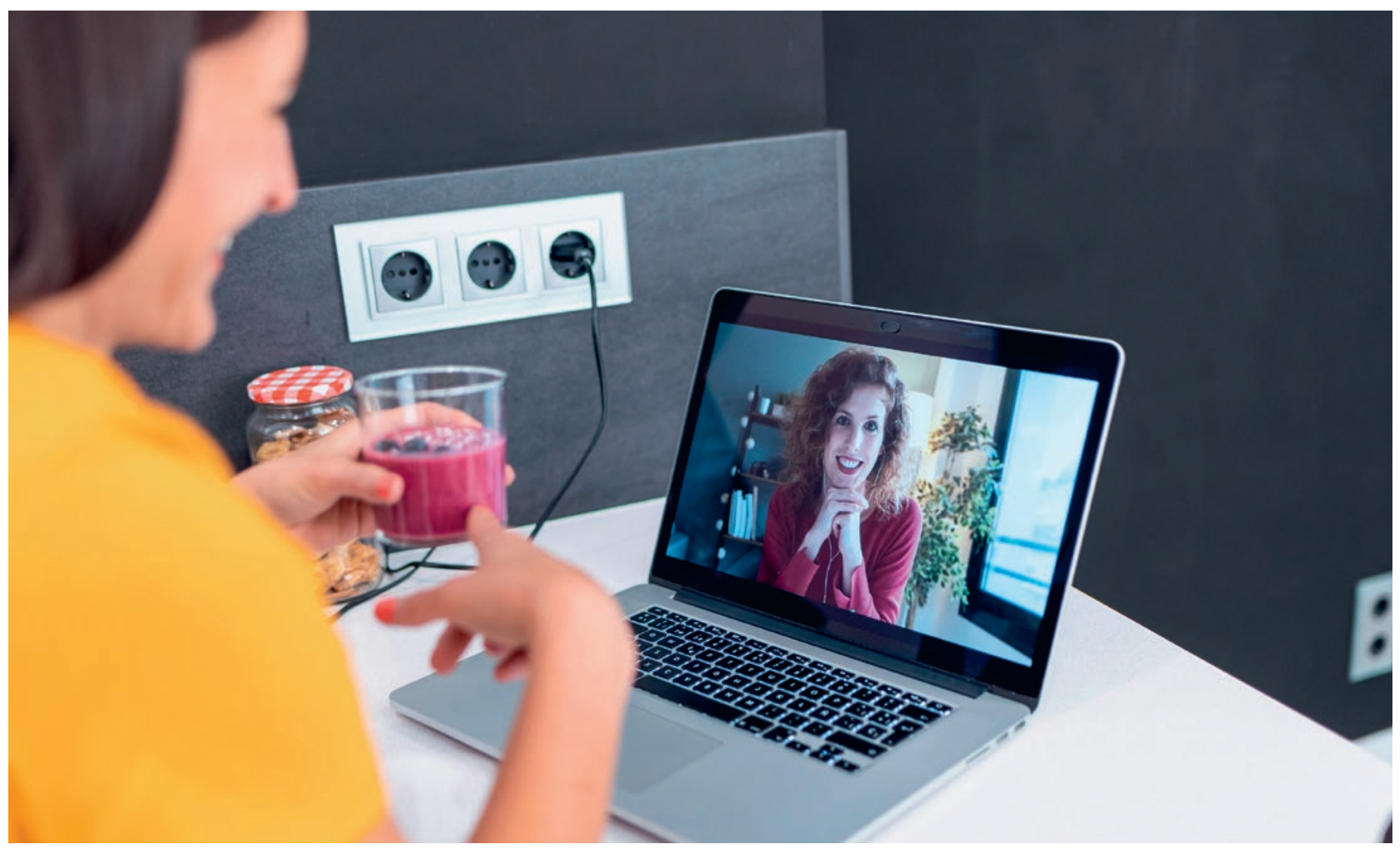

Viele Patienten wünschen sich, ergänzend zur klassischen Ernährungsberatung vor Ort, eine Fernberatung.

\title{
Interprofessionell und digital
}

\section{Andrea Sutter ${ }^{a, b}$, Beat Rickenbacher ${ }^{c}$, Carmen Binder ${ }^{b, d}$}

${ }^{a}$ BSc BFH Ernährung \& Diätetik, Leitung Digitale Ernährungsberatung; ${ }^{b}$ Oviva, Altendorf; ${ }^{\circ}$ Dr. med., Facharzt für Allgemeine Innere Medizin, Hausarztzentrum am Stadtweg, Rheinfelden; ${ }^{d}$ med. pract., MSc, Medical Director

Aufgrund der steigenden Zahl chronisch Kranker sind neue Ansätze gefragt, um diese Menschen möglichst effizient und effektiv zu betreuen. Dabei spielen interprofessionelle Zusammenarbeiten und technologische Möglichkeiten eine wichtige Rolle. Ein Beispiel dazu ist die technologisch unterstützte Ernährungsberatung, integriert in die lokale Arztpraxis.

\section{Die Herausforderungen in der Betreuung chronisch Kranker}

Weltweit nimmt die Zahl der chronischen Erkrankungen stetig zu [1]. 2012 machten sie weltweit bereits über zwei Drittel der Todesfälle aus und führten zu hohen menschlichen, sozialen und ökonomischen Kosten [1]. Die Schweiz bildet dabei keine Ausnahme, und WHO, OECD sowie der Bund warnen vor einer weiteren $\mathrm{Zu}$ nahme der Zahl von Patientinnen und Patienten mit chronischen Erkrankungen [2, 3]. Verhaltensbedingte
Risikofaktoren wie ungesunde Ernährung und Bewegungsmangel haben einen grossen Anteil an deren Entstehung [1, 3]. Daher sind bei chronischen Krankheiten wie Diabetes mellitus, Adipositas oder HerzKreislauf-Erkrankungen langfristige Veränderungen des Ernährungsverhaltens auch in der ersten Stufe der Therapie enthalten [2, 4-7]. Ambulante Ernährungsberatung wird klassischerweise in Ernährungsberatungspraxen oder Ambulatorien von Spitälern erbracht. Diese örtliche Distanz zur Hausarztpraxis bringt jedoch Barrieren für eine optimale Zusammenarbeit mit 
sich. Die Hausärztinnen und Hausärzte wünschen zur Förderung der Zusammenarbeit eine verbesserte Kommunikation, angepasste Präsenzzeiten, den persönlichen Kontakt zur Ernährungsberaterin und dass die Behandlung sichtbare Erfolge zeige [8]. Auch für die Patientinnen und Patienten gilt es, im klassischen Ernährungsberatungs-Setting Hürden zu überspringen, sei es der Zeitaufwand für den Weg in die Beratungspraxis oder das limitierte Terminangebot.

\section{Interprofessionelle und digitale Betreuung}

Es sind daher neue, zeitgemässe Ansätze gesucht, um die Reichweite und den Effekt von ernährungstherapeutischen Interventionen zu skalieren und die Ressourcen der Akteure optimal einzusetzen. Grundsätzlich erachten in der Schweiz sowohl die Ärzteschaft wie auch die Bevölkerung die Nutzung von digitalen Möglichkeiten in der Gesundheitsversorgung als sehr wichtig, wobei das Interesse der Bevölkerung grösser zu sein scheint als jenes der Ärzteschaft [9-11]. Diese

Sowohl die Ärzteschaft wie auch die Bevölkerung erachten die neuen digitalen Möglichkeiten im Gesundheitswesen als sehr wichtig.

sieht zwar viele Möglichkeiten von digitalen Anwendungen in der Praxis; angeboten werden die Dienstleistungen jedoch erst von einer Minderheit [11]. Die Herausforderungen für die Ärzteschaft mit ihren bisherigen Möglichkeiten sind unter anderem mangelnde Interoperabilität, fehlender Nutzennachweis oder die Erwartung neuer Datenschutzprobleme [12]. Für diese wichtigen Herausforderungen gibt es Lösungen. Datenschutz muss hoch priorisiert werden, und die Systeme müssen entsprechend gesichert sein. Die Bevölkerung scheint der Ärzteschaft im Umgang mit ihren Gesundheitsdaten mehrheitlich zu vertrauen. Digitale Angebote werden von Patientinnen und Patienten sogar zunehmend eingefordert $[9,10]$.

\section{Ernährungsberatung aus der Ferne zeigt Wirkung}

Die Literatur zeigt, dass durch Fernbetreuung bzw. hybride Ansätze mindestens ebenbürtige, wenn nicht sogar bessere Resultate als bei der traditionellen VorOrt-Beratung erwartet werden können $[8,9]$. So demonstrieren Haas et al., dass Ernährungsberatung bei Adipositas über eine App zu signifikanter Gewichtsreduktion und einer Verbesserung der metabolischen

\section{Erfahrungen mit Oviva}

Der Leistungserbringer Oviva bietet seit 2015 ein Modell zur Ernährungsberatung an, welches traditionelle Vor-Ort-Beratung mit technologisch unterstützter Fernberatung ergänzt. Die regionale «Flying Dietitian» ist je nach Bedarf zwischen einem Halbtag pro Monat bis mehrereTage pro Woche direkt in der Arztpraxis vor Ort und berät die Patienten dazwischen optional aus der Ferne mittels App, Chat, Telefon und Videobesprechung. Aktuelle Auswertungen zeigen die Motivation und Compliance der behandelten Patienten sowie steigende Nachfrage nach der digitalen Ernährungsberatung. 92\% der Patienten, welche zur Ernährungsberatung dem Leistungserbringer zugewiesen werden, erscheinen zur ersten Sitzung, und $71 \%$ der Patienten nehmen drei oder mehr Sitzungen in Anspruch. 2019 entschieden sich 42\% der Patienten für eine hybride Ernährungsberatung, also für die zusätzliche Unterstützung via App, was eine Steigerung von mehr als $10 \%$ zum Vorjahr bedeutet. Das hybride Modell der Ernährungsberatung mit Vor-Ort- und Fernbetreuung wird inzwischen in über 350 Arztpraxen in der Schweiz angeboten.

Parameter führen kann [8]. Der mediane Gewichtsverlust sowie die Blutdruckreduktion über den Zeitraum eines Jahres waren signifikant $(-4,9 \mathrm{~kg}$ bzw. $-6 \%$ des Körpergewichtes und $-11,2 \mathrm{~mm} \mathrm{Hg}$ [systolisch] respektive $-5,5 \mathrm{~mm} \mathrm{Hg}$ [diastolisch]). Ausserdem wurde eine signifikante Abnahme des Körperfetts und des Bauchumfangs verzeichnet. Auch Veränderungen im Essverhalten wie ein vermehrter Gemüse- und Früchteverzehr bzw. eine Reduktion des Alkohol-, Süssigkeiten- und Fettkonsums konnten festgestellt werden [13]. Beim Direktvergleich von traditioneller Vor-Ort-Beratung und Fernberatung zeigten Appel et al. bei beiden Gruppen eine signifikante Gewichtsveränderung im Vergleich zur Kontrollgruppe [14]. Johnson et al. zeigten mit dem Fernbetreuungsmodell via Videotelefonie gar bessere Resultate bei Gewichtsverlust, Aktivitätslevel

Langfristige Verhaltensänderungen werden durch häufige Repetition des neuen Verhaltens über die Zeit herbeigeführt.

und metabolischen Markern [15]. Vermutlich führt ein Teil des Erfolges auf die Tatsache zurück, dass Verhaltensänderungen durch häufige Repetition des neuen Verhaltens über die Zeit herbeigeführt werden müssen [16]. Durch hochfrequente Beratungseinheiten aus der Ferne und somit minimalen Aufwand für den Patienten kann dies optimal unterstützt werden [17]. Ausserdem ist bekannt, dass Betroffene, die Apps für ihr Krankheitsmanagement nutzen, im Allgemeinen eine positive Korrelation mit der Selbstfürsorge zeigen [18].

Die Oviva AG bietet seit 2015 ein Modell zur Ernährungsberatung an, welches traditionelle Beratung vor Ort mit technologisch unterstützter Fernberatung ergänzt. 


\section{Literatur}

1 WHO, Global status report on noncommunicable diseases 2014. 2014: p. 298.

2 GDK, B.u., Nationale Strategie zur Prävention nichtübertragbare Krankheiten. 2016

3 OECD and W.H. Organization, OECD-Berichte über Gesundheitssysteme: Schweiz 2011. 2012.

4 Kurt Laederach, D.D., Philipp Gerber, Zoltan Pataky. Consensus du traitement de l'obésité 2016. 2016.

5 Felix Huber, U.B., Andrea Rosemann. Medix Guideline Hyperlipidämie. 2016.

6 Roger Lehmann, G.G., Astrid Czock, Marc Egli, Doris FischerTaeschler, Markus Laimer, Barbara Lucchini, Sebastien Thalmann, Peter Wiesli. Swiss Recommendations of the Society for Endocrinology and Diabetes (SGED/SSED) for the Treatment of Type 2 Diabetes Mellitus (2020). 2020.

7 Bryan Williams, G.M., Wilko Spiering, Enrico Agabiti Rosei, Michel Azizi, Michel Burnier, Denis L. Clement, Antonio Coca, Giovanni de Simone, Anna Dominiczak, Thomas Kahan, Felix Mahfoud, Josep Redon, Luis Ruilope, Alberto Zanchettit, Mary Kerins, Sverre E. Kjeldsen, Reinhold Kreutz, Stephane Laurent, Gregory Y. H. Lip Richard McManus, F. R. Krzysztof Narkiewicz, Roland E. Schmieder, Evgeny Shlyakhto, Costas Tsioufis, Victor Aboyans, Ileana Desormais. 2018 ESC/ESH Guidelines for the management of arterial hypertension. 2018.

8 Daniela Bösiger, S.K. Optimierung der Zusammenarbeit zwischen Hausärztinnen, Hausärzten und Ernährungsberaterinnen, -beratern - Für eine qualitativ hochstehende Patientenbetreuung. 2016.

9 Fabian Röthlisberger, R.S. Digital Trends Survey 2019. Schweiz Ärzteztg. 2020;101(19-20):622-5.

\section{Das Wichtigste in Kürze}

- Weltweit nimmt die Zahl der chronischen Erkrankungen stetig zu. Risikofaktoren wie ungesunde Ernährung und Bewegungsmangel haben einen grossen Anteil an der Entstehung chronischer Krankheiten. Veränderungen des Ernährungsverhaltens und damit das Hinzuziehen von Ernährungsberaterinnen und -beratern stehen daher oft am Anfang der Betreuung chronisch Kranker.

- Da das klassische Setting der Ernährungsberatung in spezialisierten Praxen oder Ambulatorien sowohl von Ärzte- wie auch von Patientenseite oft bemängelt wird (Kommunikation zur Hausärztin/zum Hausarzt sollte verbessert werden, Zeitaufwand für den Weg in die Beratungspraxis, limitiertes Terminangebot etc.), sind neue Betreuungsansätze gefragt.

Andrea Sutter

Oviva AG

Zürcherstrasse 64

CH-8852 Altendorf

andrea.sutter[at]oviva.com
- Laut den Autorinnen und dem Autor zeigen digitale Fernbetreuung oder hybride Ansätze, die die Betreuung vor Ort mit Fernbetreuung kombinieren, mindestens ebenbürtige, zuweilen bessere Resultate als klassische Behandlungen.
10 FMH, Digital Trends Survey 2019. 2020.

11 Tarja Zingg, R.S., Fabian Röthlisberger. Digitalisierung in der ambulanten Gesundheitsversorgung. Schweiz Ärzteztg. 2019;100(5):113-6.

12 Kong T, et al. Physician attitudes towards - and adoption of - mobile health. Digit Health. 2020;6:2055207620907187.

13 Haas K, Hayoz S, Maurer-Wiesner S. Effectiveness and Feasibility of a Remote Lifestyle Intervention by Dietitians for Overweight and Obese Adults: Pilot Study. JMIR Mhealth Uhealth. 2019;7(4):e12289.

14 Appel LJ, et al. Comparative effectiveness of weight-loss interventions in clinical practice. N Engl J Med. 2011;365(21):195968.

15 Johnson KE, et al. Telemedicine-Based Health Coaching Is Effective for Inducing Weight Loss and Improving Metabolic Markers. Telemedicine journal and e-health: the official journal of the American Telemedicine Association. 2019;25(2):85-92.

16 Wendy Wood, D.T.N. Healthy through habit: Interventions for initiating \& maintaining health behavior change. Behavioral Science \& Policy. 2016;2(1):71-83.

17 Digenio AG, et al. Comparison of methods for delivering a lifestyle modification program for obese patients: a randomized trial. Ann Intern Med. 2009;150(4):255-62.

18 Kebede MM, Pischke CR. Popular Diabetes Apps and the Impact of Diabetes App Use on Self-Care Behaviour: A Survey Among the Digital Community of Persons With Diabetes on Social Media. Frontiers in Endocrinology. 2019;10(135).

Bildnachweis

(c) Nenitorx | Dreamstime.com (Symbolbild)

\section{L'essentiel en bref}

- Le nombre de maladies chroniques est en constante aug mentation dans le monde entier. Les facteurs de risque tels qu'une mauvaise alimentation et le manque d'exercice jouent un rôle majeur dans leur développement. La modification du comportement nutritionnel et donc la consultation de nutritionnistes marquent de ce fait souvent la première étape de la prise en charge des malades chroniques.

- Comme le cadre classique du conseil nutritionnel dans les cabinets spécialisés ou les cliniques ambulatoires est souvent critiqué, tant par les médecins que les patients (qualité de la communication avec le médecin de famille, temps de trajet jusqu'au cabinet, possibilités limitées de rendez-vous, etc.) de nouvelles formes de prise en charge sont nécessaires.

- Selon les auteures et l'auteur, les consultations en ligne ou les approches hybrides combinant prise en charge sur place et à distance donnent des résultats au moins équivalents, voire supérieurs aux traitements classiques. 